\title{
Search for TeV transients from Coalescing Binary systems discovered in Gravity Waves by LIGO/Virgo
}

\author{
Andrew J. Smith* \\ University of Maryland, College Park \\ E-mail: asmith8@umd.edu
}

The High Altitude Water Cherenkov (HAWC) Observatory is a wide-field continuously operating gamma-ray telescope. The detector consists of an array of 300 water tanks, each containing 200kTons of purified water instrumented with 4 larger area PMTs. The observatory is located at an elevation of $4100 \mathrm{~m}$ a.s.l. near the Sierra Negra volcano in central Mexico. The combination of density, area and sensitivity gives HAWC an unprecedented threshold for air shower arrays, below $1 \mathrm{TeV}$. Unlike IACTs, the HAWC operates continuously (90\% uptime) and can observe the entire overhead sky ( $2 \mathrm{sr}$ ), making it well suited for catching rapid transients from unknown directions and times. The LIGO/Virgo collaboration has recently discovered gravity wave ?bursts? emitted from coalescing massive compact objects. Gravity-wave detectors have very poor position resolution, making follow-up observations by narrow-field telescopes challenging. We have developed a systematic method for searching the locations where the probability contours overlap the HAWC field of view for gamma-ray transients. We describe in detail our analysis procedure and will report on our follow-ups observations of LIGO/Virgo triggers for both observation runs $\mathrm{O} 1$ and $\mathrm{O} 2$ (on going at the time of abstract submission).

35th International Cosmic Ray Conference - ICRC2017

10-20 July, 2017

Bexco, Busan, Korea

${ }^{*}$ Speaker. 


\section{Introduction}

The recent discovery of gravitational radiation by the LIGO/Virgo collaboration is one of the most notable scientific discoveries of the 21 st century [1]. For nearly 100 years, the theoretical models based on Einstein's theory of General Relativity have predicted the existence of gravitational radiation, but the magnitude of its effect has been too small to detect in the laboratory. With many incremental improvements over the decades, the detection of gravitational radiation was finally achieved in 2015 by the LIGO/Virgo collaboration. The discovery was announced in the Spring of 2016 and immediately recognized as one of the greatest scientific achievements of the century. The discovery has lead to greater insight into the fundamental properties of General Relativity, but further understanding is limited by the specific knowledge of the systems emitting the radiation. To this end, there is a concerted effort to organize a community of astrophysical observatories to rapidly follow-up gravitational wave triggers in an effort to identify and study the progenitors at both many wavelengths and with multiple messenger particles (gamma-rays and neutrinos).

During the 2015-2016 operational run of the Advanced LIGO instrument (referred to as O1), two high confidence gravitational wave events were detected. The first was GW150914, detected near the beginning of the operational periods and the second was GW151226, the so called "Boxing Day" event. Both of these events were reported to the greater scientific community only well after their occurrence so that appropriate checks could be made to the data to verify that they were indeed detecting a new type of radiation. As a result, the community of astrophysical observers were unable to rapidly make follow-up observations to search for the progenitors.

On November 30, 2016 the LIGO/Virgo collaboration began its second operations run (O2). During $\mathrm{O} 2$, the process for rapidly verifying the locations of gravitational wave candidates was greatly improved and a private communications channel was set up to collaborations of observers within the astrophysics community. Observatories of all types can receive real-time information about gravitational wave candidates and make their follow-ups available within the community, but they cannot share the information beyond their collaborations until the embargo is lifted. The system is administrated using the Gamma-ray burst Coordinates Network (GNC) system which was created to rapidly disseminate Gamma-Ray Burst (GRB) locations to the astrophysical community. LIGO/Virgo notifications are sent using a private GCN channel that is only accessible partner observers which have signed an MOU.

In this paper, we describe the follow-up methodology by the High Altitude Water Cherenkov (HAWC) Observatory, a wide-field gamma-ray observatory with sensitivity in the Very High Energy (VHE) band. In Section 2, we describe the challenge and potential for VHE follow-ups using HAWC, in section 3 we describe the sensitivity of HAWC to VHE transients, in section 4 we describe the results of the $\mathrm{O} 2$ run at the time of the writing, in section 5 we describe the HAWC follow-up procedure and in section 6 we describe the HAWC results for GW010417, a gravitational wave event which had its embargo lifted just prior to the writing of this paper.

\section{The Challenge and Potential of Gravitational Wave Follow-ups}

During O1, the LIGO/Virgo collaboration reported two high confidence gravitational wave 
events, GW150914 and GW151226. Both of these were identified based on their time structure as being due to the coalescence of two medium sized black holes. The former had total mass of $62 \mathrm{M}_{\text {Solar }}$ and the latter $22 \mathrm{M}_{\text {Solar }}$. In both cases the masses of the black holes were found to be similar. The total energy radiated in gravitational radiation in the first event was an astounding 3 $\mathbf{M}_{\text {Solar }}$ and in the second event was $1 \mathbf{M}_{\text {Solar }}$. The distance to these objects was estimated to be roughly $400-500 \mathrm{Mpc}$, which corresponds to a redshift of 0.09 . A third event has was recently reported. The details of this third event are described in section 6 .

One major challenge for following up gravitational wave triggers is that even for highly significant detections, the location of the event on the sky can not be well isolated. For example the highly significant GW151226 event has an 90\% error contour on the sky that subtended XXX square degrees (figure 1). Furthermore, the contour generally follows a great circle and cannot be fully observed by a single follow-up instrument simply due to occultation by the earth. As a result, follow-up instruments with wide fields of view and high duty cycles are preferred.

For VHE gamma-ray observations signal attenuation can significant reduce an instrument's sensitivity to objects at cosmological distances through pair-production on the inter-galactic radiation fields. This attenuation can block gamma rays with energies greater than a few hundred $\mathrm{GeV}$ for source at a distance greater than $\mathrm{z}=0.09$, the distance to the first two gravitational wave sources. Additionally, it has been theorized that $\mathrm{BH}-\mathrm{BH}$ mergers might be almost entirely matter and radiation free events, and therefore have almost no electromagnetic or neutrino counterpart. The more favorable target for EM follow-ups are the NS-NS or NS-BH merger channels. These events may contain significant matter and radiation fields capable of being observed in gamma-rays and other bands. In contrast, the nominal horizon for NS-NS events is only about 70Mpc, which is sufficiently close that $100 \mathrm{GeV}-10 \mathrm{TeV}$ radiation can readily reach the Earth. While HAWC is probably unable to view the most distant events, the most promising events for gamma-ray follow up should be easily visible to HAWC should they emit.

\section{The HAWC Detector}

The High Altitude Water Cherenkov (HAWC) observatory is a large-area VHE gamma-ray detector located at an elevation of 4100m (13450 ft) at the Sierra Negra Volcano in Central Mexico. The detector itself covers an area of roughly $22,000 \mathrm{~m}^{2}$ with an array of 300 modular water tanks (see figure 2). Each tank measures $7.3 \mathrm{~m}$ in diameter and has a height of $5 \mathrm{~m}$. The tanks are filled to a depth of $4.5 \mathrm{~m}$ with purified water. Collectively, the array of tanks holds more than 50 ktons of water. Each tank is instrumented with on the bottom of the tanks are 4 upward-facing Photomultiplier Tubes (PMTs). High-energy particles from extensive air showers in the atmosphere that penetrate to the observation level are detected through the Cherenkov radiation that they produce in the water volume. The detector can effectively reconstruct cascades produced by Very High Energy (VHE) cosmic rays and gamma rays impinging on the atmosphere with an effective array of 100 $\mathrm{m}^{2}$ at $100 \mathrm{GeV}$ that rapidly increases with energy and plateaus where the effective area matches the physical area at $1 \mathrm{TeV}$.

Unlike pointed instruments, HAWC can observe the entire overhead sky with an energy threshold that gradually increases with zenith angle. The field of view of HAWC is approximately $2 \mathrm{sr}$ $\left(0-45^{\circ}\right.$ zenith angle range) where the central steradian $\left(0-30^{\circ}\right.$ zenith angle range $)$ is more sensitive. 


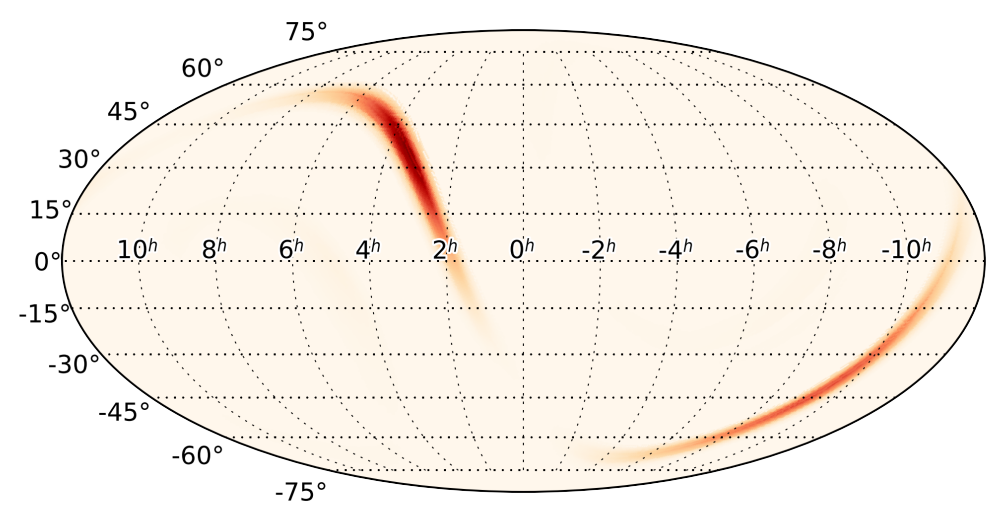

Figure 1: Sky localization contour for gravitational wave event GW151226.

At any given instant, HAWC is observing about $16 \%$ of the sky and with the rotation of the earth, can survey roughly $2 / 3$ of the sky over the course of a day. HAWC is able to operate both day and night and generally has an uptime greater than $90 \%$ with downtime almost entirely due to power failures or system maintenance. Operation does not depend on consumables and astrophysical observations do not need to cease to perform instrument calibrations. Triggers are formed when 30 PMTs are hit within a 150ns window. HAWC triggers at a rate of $25 \mathrm{kHz}$ which is dominated by air showers produced by charged cosmic-ray hadrons.

The sensitivity of the HAWC detector was established through both Monte Carlo simulations and observations of the Crab plerion [2]. A good rule of thumb is that HAWC detects the Crab at roughly $5 \sigma$ with each transit of roughly 5 hours. At the other extreme, very short duration emission, HAWC has searched for GRBs both coincident with satellite observations [3] and through an unguided search [4]. We estimate that HAWC is sensitive to the most fluent GRBs, which only occur annually or less [7]. In 2 years of operation, we have not detected a GRB, but we continue to look. Note however, that since GRBs are overwhelmingly at large redshifts, the main factor that limits HAWC's sensitivity is the attenuation of the VHE gamma-rays in transit, not the emission itself. As gravitational wave are anticipated to be much closer than gamma-ray bursts HAWC may very well be sensitive to the VHE emission granted that it is produced. Figure 4 shows the sensitivity of HAWC as a function of zenith angle to short GRBs (1s) for various redshifts.

\section{O2 Run by LIGO/Virgo}

For the second observing period (O2), the LIGO/Virgo collaboration releases information only 


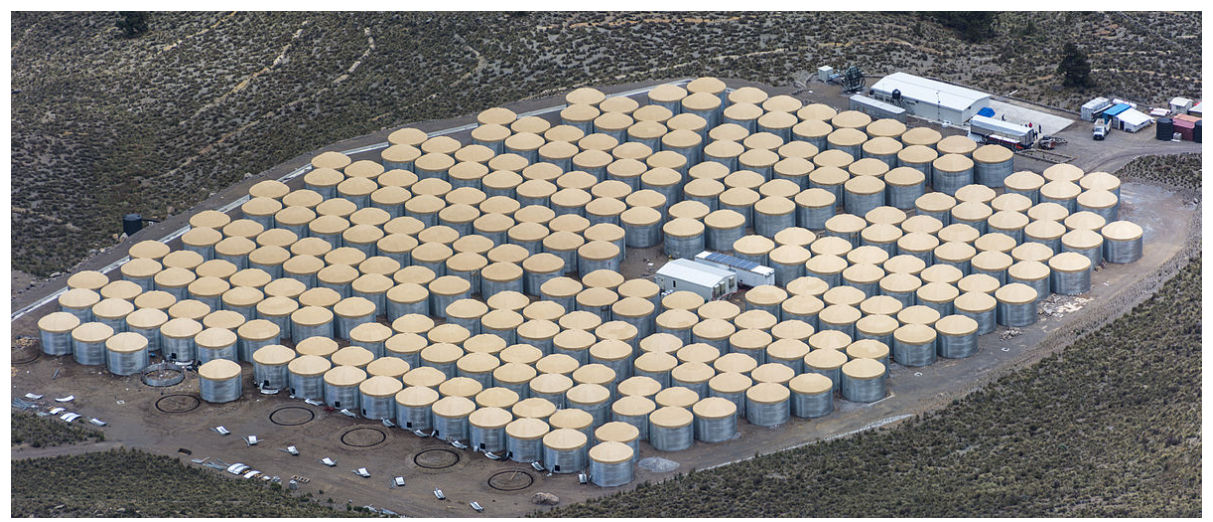

Figure 2: Image of the HAWC Detector.

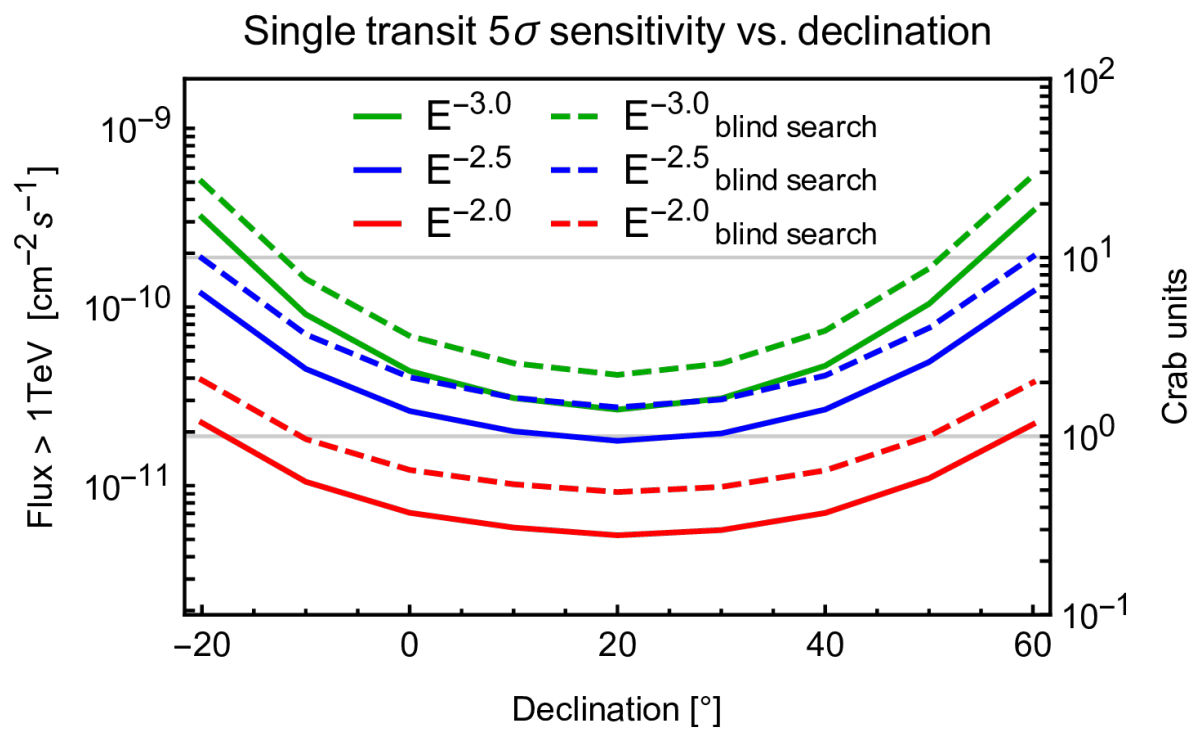

Figure 3: Sensitivity of HAWC to a transiting source for a single transit as a function of declination.

to MOU partners with the purpose of facilitating rapid follow-up observations. The information released is strictly embargoed as the results are often preliminary and may be unverified. The LIGO/Virgo Collaboration has publicly reported from November 30, 2016 to May 3, 2017 the 67 days if Hanford-Livingston coincident science data have been collected. During this period, 6 triggers have been issued to MOU partners with a false-alarm threshold (FAR) of one per month. LIGO reports an average reach of $70 \mathrm{Mpc}$ for $1.4 \mathrm{M}_{\text {Sun }}+1.4 \mathrm{M}_{\text {Sun }}$ mergers, $300 \mathrm{Mpc}$ for $10 \mathrm{M}_{\text {Sun }}+$ $10 \mathrm{M}_{\text {Sun }}$ and $700 \mathrm{Mpc}$ for $30 \mathrm{M}_{\text {Sun }}+30 \mathrm{M}_{\text {Sun }}$ events. For more details on LIGO's on-going release of data, see their public web page [5].

\section{Gravity Wave Event Follow-up procedure}

Upon receiving a notification of a LIGO/Virgo trigger, a small team within the HAWC col- 


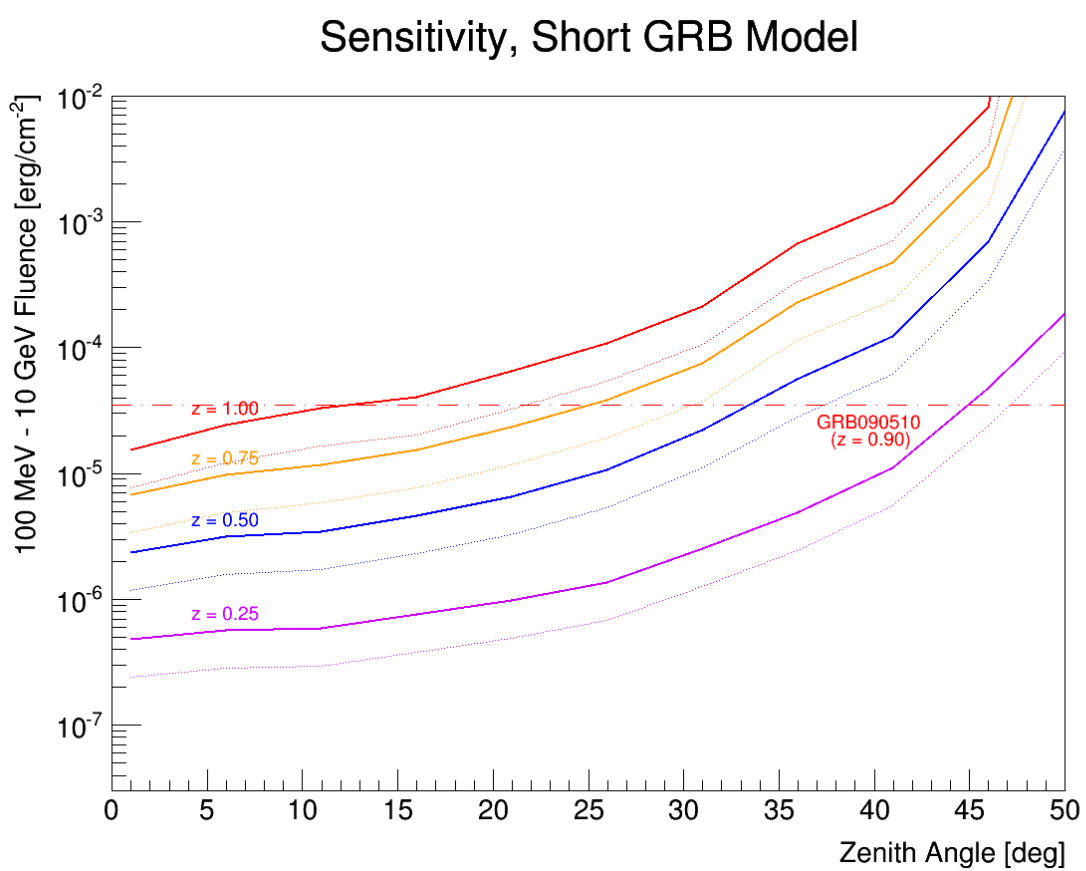

Figure 4: Shown is the MeV-GeV fluence of short duration GRBs detectable by HAWC plotted vs redshift and zenith angle. The short GRB model assumes a $1 \mathrm{sec}$ duration, $\mathrm{E}^{-} 1.6$ spectral extrapolation from lower energy. Y-axis is the fluence needed for a 5 sigma detection $50 \%$ of the time. Solid line indicates sensitivity after accounting for 1 year of trials. Dashed line marks single-trial sensitivity relevant to triggered searches.

laboration is immediately notified and a multi-step procedure is followed to verify and analyze the data in real time to produce a rapid response to the observer community. The procedure is based on two well tested gamma-ray search algorithms, a long durations (hours to years) algorithm that is optimized to detect crab-like sources [6] and a short duration algorithm that is used to search for emission from 0.1s to 100s and is optimized for the detection of distant GRBs [4].

Procedure:

- Verify that HAWC is currently operational and notify shift personnel to keep the experiment operational for $24 \mathrm{hrs}$. This may mean postponing maintenance or other servicing.

- Burst Search - Quick Look: We check results of automatic realtime GRB search algorithm's for significant results that could be coincident with the gravitational wave event. This system searches for anomalous excesses in bins overlapping in space and time at time scales of $0.1 \mathrm{~s}$, $1 \mathrm{~s}, 10 \mathrm{~s}$ and $100 \mathrm{~s}$. Due to the large number of trials (order $10^{18}$ in a year of searches), the blind search has a high intrinsic pre-trials threshold of about $10 \sigma$. For details on this search see [4].

- Burst Search - Reduced Threshold Follow-up: We lower the threshold and search for prompt gamma-ray emission near the time of the gravitational wave trigger. We run the search algorithm with a reduced reporting threshold on data collected around the time of the trigger. We search for emission with start times ranging from $T_{0}-\Delta T$ to $T_{0}+5 \Delta T$, where $T_{0}$ is the 
time of the trigger and $\Delta T$ is the duration of the time bin being searched. As before, the search bins overlap in space and time. Time scales from $0.1 \mathrm{~s}$ to $100 \mathrm{~s}$ are searched. Since the time window scales with $\Delta T$, there is an equal number of trials at each time scale. We identify points that have greater than $5 \sigma$ pre-trials significance that overlap with the LIGO/Virgo contours.

- Long Duration Search - Quick Look: We check the results from an online all-sky search algorithm that searches for emission from transiting sources. As portions of the contour that pass through HAWC's field-of-view set below HAWC's effective horizon, the emission is computed and excesses or upper limits are calculated. This program runs continuously and reports all setting points with a pre-trials significance greater than $5 \sigma$.

- Long Duration Search - Contour Driven Follow-up: For portions of the LIGO/Virgo contour that are in the HAWC field-of-view at the time of the trigger, we run a custom version of the 1-transit map generation, but instead of integrating over the complete transit, we only consider data collected after the time of the trigger. In this way background from the time prior to the trigger is excluded and sensitivity is increased.

- Follow-up on points identified by other observers: In some cases other observers have identified possible coincident excesses. We work to follow up these observations to see if HAWC sees anything coincident in space and time with their observations.

At the completion of this procedure, a GCN notice is drafted, approved internally and sent to the community through the private channel set up for LIGO/Virgo.

\section{Results from January 4, 2017 Event}

The LIGO/Virgo collaboration has lifted the embargo on one of the events, GW170104. This is a high confidence detection that, as the two prior events, has a $\mathrm{BH}-\mathrm{BH}$ coalesces as its progenitor. Figure 5 shows the LIGO/Virgo contour and the HAWC field of view at the time of the trigger. For this event, a significant portion of the contour was within HAWC's field of view. We searched for emission on time scales from 0.1 s to 1 transit and found nothing significant and followed up with a GCN notice within a few days of the original notice.

\section{Conclusion}

During observation run 2 of the Advanced LIGO instrument, HAWC has a detailed plan for following up GW events and has actively participated in the private communication process in the observer community. After the completion of O2, the LIGO/Virgo collaboration will lift the embargo on the triggers and we will make public our findings on all the GW triggers.

\section{Acknowledgments}

We acknowledge the support from: the US National Science Foundation (NSF); the US Department of Energy Office of High-Energy Physics; the Laboratory Directed Research and Devel- 

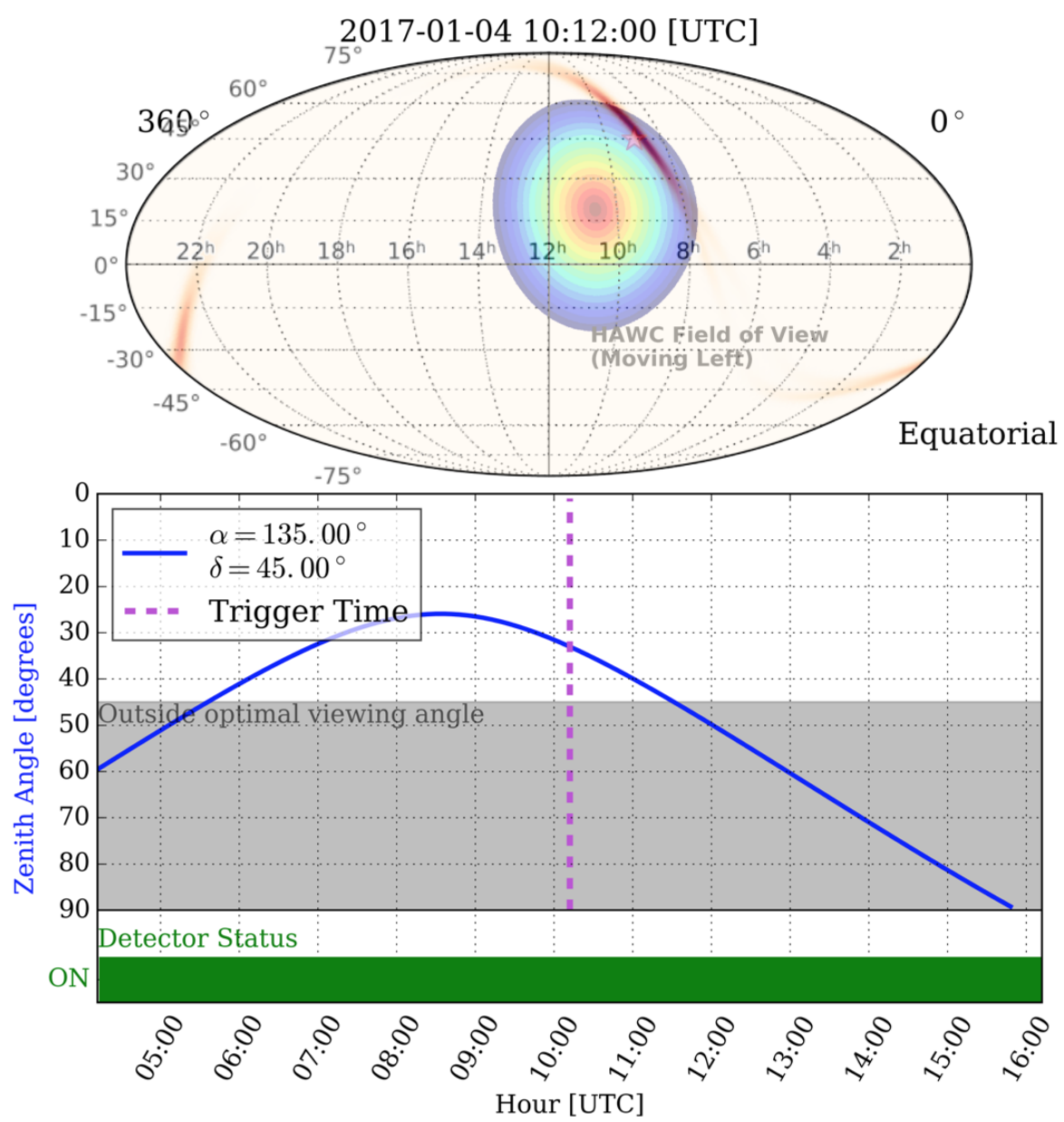

Figure 5: LIGO/Virgo contour for GW170104 and the HAWC field of view at the time of the trigger. The red shaded contour shows the gravitational wave error contour and the rainbow shaded area shows the HAWC field of view at the time of the trigger. The most probable region of the GW contour was within the HAWC field, but near the edge were the sensitivity is relatively poorer and the threshold is higher. As time progresses, the HAWC contour moves to the left, so the main northern component of the GW contour is seen as setting. This is what is indicated by the bottom figure, which shows the zenith angle of the position of the star as a function of time.

opment (LDRD) program of Los Alamos National Laboratory; Consejo Nacional de Ciencia y Tecnología (CONACyT), México (grants 271051, 232656, 260378, 179588, 239762, 254964, 271737, 258865, 243290, 132197); L'OREAL Fellowship for Women in Science 2014; Red HAWC, México; DGAPA-UNAM (grants RG100414, IN111315, IN111716-3, IA102715, 109916); VIEPBUAP; PIFI 2012, 2013, PROFOCIE 2014, 2015; the University of Wisconsin Alumni Research Foundation; the Institute of Geophysics, Planetary Physics, and Signatures at Los Alamos National Laboratory; Polish Science Centre grant DEC- 2014/13/B/ST9/945; Coordinación de la Investigación Científica de la Universidad Michoacana. 


\section{References}

[1] Abbott, B., P.. et al., Observation of gravitational waves from a binary black hole merger. Phys. Rev. Lett., 116:061102, Feb 2016.

[2] A. U. Abeysekara et al, Observation of the Crab Nebula with the HAWC Gamma-Ray Observatory. ArXiv e-prints, January 2017. (accepted for publication in ApJ).

[3] R. Alfaro et al, Search for very-high-energy emission from Gamma-ray Bursts using the first 18 months of data from the HAWC Gamma-ray Observatory. ArXiv e-prints, May 2017.

[4] J. Wood, et al., Proceedings of the 2017 International Cosmic Ray Conference (This Proceedings).

[5] http://www.ligo.org/news.php

[6] Martinez, I. et al, Proceedings of the 2017 International Cosmic Ray Conference (This Proceedings).

[7] I. Taboada and R. C. Gilmore. Prospects for the detection of GRBs with HAWC. Nuclear Instruments and Methods in Physics Research A, 742:276-277, April 2014. 